\title{
The Research Progress and Application Expectation of Metal Rubber Vibration Isolator
}

\author{
Shu-lu Wang1, Hong-bai Bai1 \& Ghun-hong Lu1 \\ ${ }^{1}$ Department of Vehicles and Electrical Engineering, Mechanical Engineering College, \\ Shijiazhuang, 050003, China
}

Keywords: metal rubber, vibration isolator, engineering application, parameter identification.

\begin{abstract}
With the rapid development of science and technology, all kinds of high technical equipment is facing more and more serious problem of vibration isolation buffer under special environment. In order to adapt to this new situation, we designed the metal rubber vibration isolator. Firstly, this paper briefly describes the preparation technology of metal rubber and the research background of the metal rubber vibration isolator. In addition, this paper introduces the research progress from three aspects, including experimental study, dynamic modeling and parameter identification and research of vibration isolation system response. Finally, the future application of metal rubber vibration isolator is discussed in this paper.
\end{abstract}

\section{Introduction}

Metal rubber material is a new elastic damping material, which is manufactured from metal wire through the process of winding, stretching, laying and damping. It has the high elasticity and large damping characteristics of rubber, meanwhile, it has the excellent performance of metal materials such as high/low temperature resistance, corrosion resistance, not easy to aging, radiation resistance, less volatile in vacuum and long-term preservation. Metal rubber components can be used as alone as a vibration isolation components, and it can also be made into various kinds of metal rubber vibration isolators by adding metal cases. Inside the metal rubber is space grid structure formed by metal wire's mutual crisscross and interconnection. When under cyclic loading, friction, slipping, extrusion and deformation occurs inside the material between turns, which consumes a large amount of vibration energy, thus has the effect of damping vibration [1]. Preparation of metal rubber is shown in figure 1.

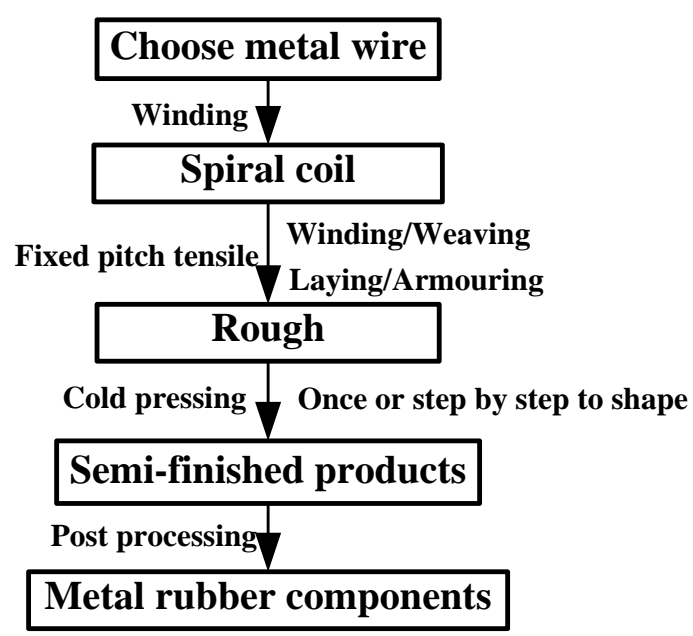

Fig.1: Metal rubber preparation process flow

\section{Research of metal rubber vibration isolator}

In the 1960s, the U.S. military first used metal rubber in military aircraft airborne equipment for vibration reduction and buffer. Since the 1970s, the experts of Russia Samar, national university of aeronautics and astronautics have carried on sustainable theory and experimental research of metal 
rubber which is intercepted from the U.S. plane. So far, the research achievements of Samar, national university of aeronautics and astronautics is in the world leading level. Russia's space manned spacecraft re-entry capsule and military aircraft su-27 all used metal rubber material damper for vibration reduction [2]. Until the 1990s, Chinese scholars came into contact with the metal rubber material and carried out the corresponding theoretical and experimental research. Due to its superior performance, the metal rubber vibration isolator can solve the problem of vibration isolation under extreme environment. Its stable performance and long service life has a practical significance to strengthen the maintainability and improve the reliability of the equipment even under normal environment [3].

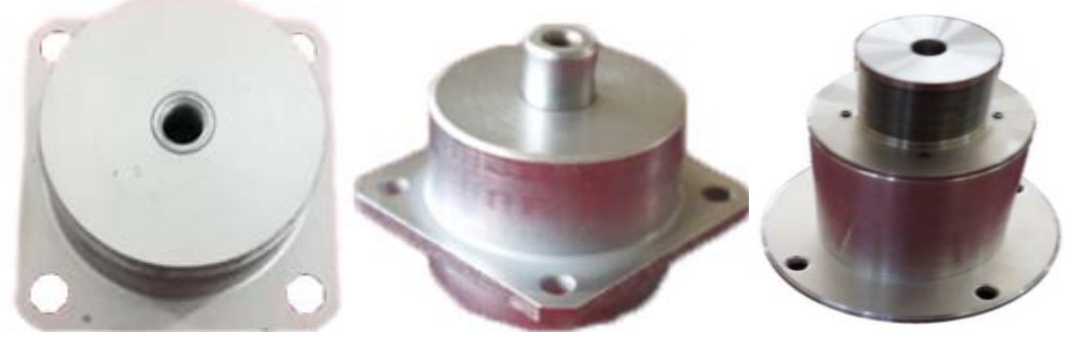

Fig.2: Several kinds of metal rubber vibration isolators

\section{Experimental study}

Chen Liang carried out the fatigue life test of the metal rubber vibration isolator with the method of control stress, finding that with the increase of vibration frequency, the resonance frequencies, resonance transmission rate and energy dissipation coefficient of the metal rubber vibration isolator damps. When the specified vibration isolator's resonance frequency decays to $90 \%$ of its maximum when the corresponding vibration frequency is just its fatigue life, he obtained fatigue life experience formula of vibration isolator from the experimental data. We can characterize the fatigue life of the metal rubber vibration isolator with the vibration frequency $\mathrm{N}$. The vibration frequency $\mathrm{N}$ can be calculated by the following formulas: $N=\sum f_{i} t_{i}, f_{i}, t_{i}$-the i-th excitation frequency and time

We considered the four factors in the fatigue life test, including relative density, wire diameter, prestressing force, and working stress. Finally, we derived the relationships between the fatigue life and each factors.

$$
\left\{\begin{array}{l}
b=7.767 \times 10^{-6} \sigma_{Q}^{-3.34}+0.01852 \\
k_{1}=8.455 \times 10^{4} \bar{\rho}^{7.494} \\
k_{2}=4.292 d_{W}+0.4849 \\
\sigma_{\max }=1.692 e^{-b\left(\log N-\log \left(k_{1} k_{2}\right)\right)^{2}} \\
N=k_{1} k_{2} 10^{\sqrt{\ln \left(1.692 / \sigma_{\max }\right) / b}}
\end{array}\right.
$$

$\bar{\rho}$-relative density, $d_{W}$-wire diameter, $\sigma_{Q}$-prestressing force, $\sigma_{\max }$-working stress

\section{Dynamic modeling and parameter identification}

Yan Hui, Jiang Hongyuan etc adoped the research method of combining theory with practice. They started with single degree of freedom situation, and built a dynamic model of metal rubber vibration isolator of aeroengine external pipeline system in which the parameters have physical meaning [4]. According to the function test of single degree of freedom, they studied the experimental identification method of relevant parameters, and they translated nonlinear equations into linear equations about parameter by using energy method and the least square method, thus identificated the parameters of the metal rubber vibration isolator. The identification results have 
good consistency with the experimental results, and the recognition results also show the influence of structure parameters on the vibration isolation performance.

Based on the metal rubber vibration isolator with base excitation, we established the mathematical model of metal rubber. The simplified mechanical model of metal rubber isolation system is shown in figure 3. Set the quality of the damping device for $m$, the based excitation was a sine signal $u(t)=u_{0} \sin w t, z(t)$ was the output displacement after the vibration isolator. By analyzing the stress of the system and Newton's second law $\sum F=m a$, we listed the movement differential equation of the system:

$$
m \ddot{z}+k(z-u)+\beta(z-u)^{3}+c(\dot{z}-\dot{u})+Y(t)=0
$$

$(z-u)$ is the relative displacement between quality and foundation.

$$
\begin{gathered}
x=(z-u) \\
m \ddot{x}+k x+\beta x^{3}+c \dot{x}+Y(t)=m u_{0} w^{2} \sin w t
\end{gathered}
$$

The overall resilience of the system is:

$$
g(t)=k x+\beta x^{3}+c \dot{X}+Y(t)
$$

In the formula, $k$-linear coefficient of spring, $\beta$-nonlinear coefficient of spring, $c$-viscous damping coefficient, $Y(t)$-The dry friction force in the process of deformation of metal rubber.

The incremental form of mathematical expressions of dry friction is:

$$
d Y(t)=\frac{k_{s}}{2}\left[1+\operatorname{sgn}\left(Y_{s}-|Y(t)|\right)\right] d x(t)
$$

The motion equation of metal rubber vibration isolator is a second order nonlinear differential equation, and $k, \beta, c, k_{s}$, and $x_{s}$ are unknown parameters. We applied energy method and the least-squares method for vibration isolator's parameters identification.

Assuming that figure 4 is the relative displacement curve $x(t)$, take half wave period $\left[t_{1}, t_{2}\right]$ :

$$
\left\{x(t) \mid t \in\left[t_{1}, t_{2}\right], x_{1}(t)=x_{2}(t)=0\right\}
$$

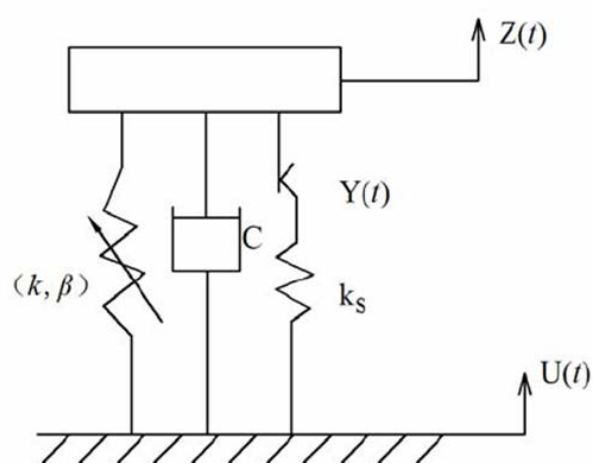

Fig.3: Several kinds of metal rubber vibration isolator

The elastic restoring force $g_{1}(t)=k x+\beta x^{3}$, its integral results has nothing to do with the path integral.

$$
\begin{gathered}
x_{1}(t)=x_{2}(t)=0 \text {, so the energy of elastic restoring force on the half wave is zero: } \\
w_{g 1}=\int_{x_{1}(t)}^{x_{2}(t)} g_{1}(t) d x=0
\end{gathered}
$$




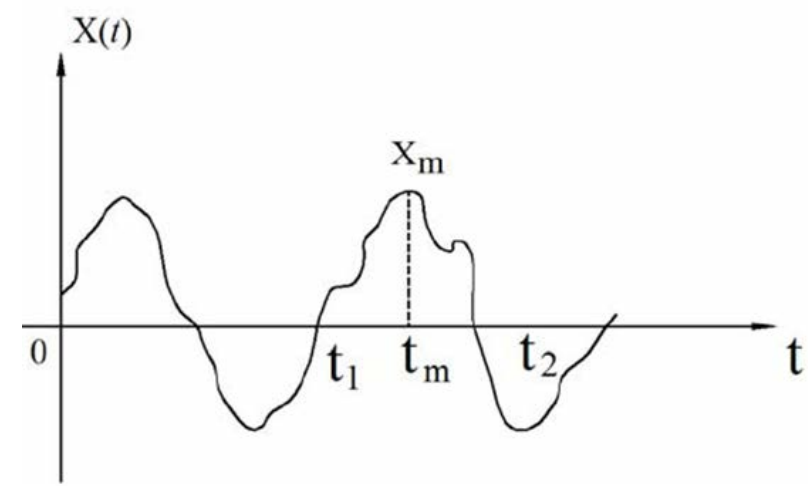

Fig.4: Several kinds of metal rubber vibration isolator

The energy of viscous damping restoring force on the half wave is:

$$
\begin{aligned}
w_{g 2}=\int_{x_{1}(t)}^{x_{2}(t)} g_{2}(t) d x=\int_{x_{1}(t)}^{x_{2}(t)} c \dot{x} d x=\int_{t_{1}}^{t_{2}}(c \dot{x})^{2} d t=c v \\
v=\int_{t_{1}}^{t_{2}}(\dot{x})^{2} d t>0
\end{aligned}
$$

The energy of dry friction link on the half wave is:

$$
w_{g 3}=\int_{x_{1}(t)}^{x_{2}(t)} g_{3}(t) d x=2 \int_{x_{s}}^{x_{m}} Y_{s} d x=2 \int_{x_{s}}^{x_{m}} k_{s} x_{s} d x=2 k_{s} x_{s}\left(x_{m}-x_{s}\right)
$$

The energy of system overall resilience on the half wave is the sum of each element energy dissipation:

$$
w_{g}=\int_{x_{1}(t)}^{x_{2}(t)} g(t) d x=\sum_{i=1}^{3} w_{g i}=c v+2 k_{s} x_{s}\left(x_{m}-x_{s}\right)
$$

According $g(t)=k x+\beta x^{3}+c \dot{x}+Y(t)$,we can list linear equations of unknown parameters:

Written in matrix form:

$$
\left\{\begin{array}{c}
c v(1)+2 k_{s} x_{s} x_{m}(1)-2 k_{s} x_{s}^{2}=w_{g}(1) \\
c v(2)+2 k_{s} x_{s} x_{m}(2)-2 k_{s} x_{s}^{2}=w_{g}(2) \\
\vdots \\
c v(n)+2 k_{s} x_{s} x_{m}(n)-2 k_{s} x_{s}^{2}=w_{g}(n)
\end{array}\right.
$$

We get the equation: $A a=W_{1}$

$$
\begin{gathered}
\left(\begin{array}{ccc}
c v(1) & 2 x_{m}(1) & -2 \\
c v(2) & 2 x_{m}(2) & -2 \\
\vdots & \vdots & \vdots \\
c v(n) & 2 x_{m}(n) & -2
\end{array}\right)\left(\begin{array}{c}
c \\
k_{s} x_{s} \\
k_{s} x_{s}^{2}
\end{array}\right)=\left(\begin{array}{c}
w_{g}(1) \\
w_{g}(2) \\
\vdots \\
w_{g}(n)
\end{array}\right) \\
\text { Set } A=\left(\begin{array}{ccc}
c v(1) & 2 x_{m}(1) & -2 \\
c v(2) & 2 x_{m}(2) & -2 \\
\vdots & \vdots & \vdots \\
c v(n) & 2 x_{m}(n) & -2
\end{array}\right), \quad W_{1}=\left(\begin{array}{c}
w_{g}(1) \\
w_{g}(2) \\
\vdots \\
w_{g}(n)
\end{array}\right), \quad a=\left(\begin{array}{c}
c \\
k_{s} x_{s} \\
k_{s} x_{s}^{2}
\end{array}\right)
\end{gathered}
$$

According to the least-square method of parameter identification, we can calculate the least squares estimate of parameter vectors $\tilde{a}=\left(A^{T} A\right)^{-1}\left(A^{T} W_{1}\right)$

Solutions the parameter estimator of energy dissipation components:

$\tilde{c}=\tilde{a}(1), \tilde{k}_{s}=\tilde{a}(2)^{2} / \tilde{a}(3), \tilde{x}_{s}=\tilde{a}(3) / \tilde{a}(2)$

Residual error estimate is: $\varepsilon=W_{1}-A \tilde{a}$, Correlation coefficient is: 


$$
p_{1}^{2}=1-\frac{\varepsilon_{1}^{T} \varepsilon_{1}}{W_{1}^{T} W_{1}}=\frac{\left(W_{1}^{T} A\right)\left(A^{T} A\right)^{-1}\left(A^{T} W\right)}{W_{1}^{T} W_{1}}
$$

Through the parameter identification we get conservative restoring force:

$$
g_{1}(t)=g(t)-\tilde{c} \dot{x}-\tilde{Y}(t)=k x-\beta x^{3}
$$

And then identify the parameters of conservative elements. The sum of the energy conservative elements stored is:

$$
\widehat{w}=w_{k}+w_{\beta}=\int_{x\left(t_{1}\right)}^{x\left(t_{m}\right)} g_{1}(t) d x=\frac{1}{2} k x_{m}^{2}+\frac{1}{4} \beta x_{m}^{2}
$$

List the equations:

$$
\left\{\begin{array}{c}
\frac{1}{2} k x_{m}^{2}(1)+\frac{1}{4} \beta x_{m}^{4}(1)=\widehat{w}(1) \\
\frac{1}{2} k x_{m}^{2}(2)+\frac{1}{4} \beta x_{m}^{4}(2)=\widehat{w}(2) \\
\vdots \\
\frac{1}{2} k x_{m}^{2}(n)+\frac{1}{4} \beta x_{m}^{4}(n)=\widehat{w}(n)
\end{array}\right.
$$

Written in matrix form:

$$
\text { Set } B=\left(\begin{array}{cc}
\frac{1}{2} x_{m}{ }^{2}(1) & \frac{1}{4} x_{m}{ }^{4}(1) \\
\frac{1}{2} x_{m}{ }^{2}(2) & \frac{1}{4} x_{m}{ }^{4}(2) \\
\vdots & \vdots \\
\frac{1}{2} x_{m}{ }^{2}(n) & \frac{1}{4} x_{m}{ }^{4}(n)
\end{array}\right), \quad b=\left(\begin{array}{c}
k \\
\beta
\end{array}\right), \quad W_{2}=\left(\begin{array}{c}
\widehat{w}(1) \\
\widehat{w}(2) \\
\vdots \\
\widehat{w}(n)
\end{array}\right)
$$

According to the least-square method of parameter identification, we can calculate the least squares estimate of parameter vectors $\tilde{b}=\left(B^{T} B\right)^{-1}\left(B^{T} W_{2}\right)$

Residual error estimate is: $\varepsilon=W_{2}-B \tilde{b}$, Correlation coefficient is:

$$
p_{2}{ }^{2}=1-\frac{\varepsilon_{2}{ }^{T} \varepsilon_{2}}{W_{2}{ }^{T} W_{2}}=\frac{\left(W_{2}^{T} B\right)\left(B^{T} B\right)^{-1}\left(B^{T} W_{2}\right)}{W_{2}{ }^{T} W_{2}}
$$

Through the equation, we can judge the identification precision of the conservative elemen.

\section{Research of vibration isolation system response}

Zhang Jinjin deduced the frequency response equation of metal rubber vibration isolator under simple harmonic excitation by using the Fourier series expansion and harmonic balance method. She solved the frequency response equation by Matlab program and then obtained the spectrogram. She studied the effect the primary factor, three times the nonlinear factors, five times the nonlinear factors and seven times the nonlinear factors have on dynamic response of metal rubber vibration isolator separately. Studies have shown that the primary factor and three times the nonlinear factors were decisive factors for the amplitude-frequency characteristics of metal rubber vibration isolator.

\section{The engineering application}

Based on the many advantages of metal rubber isolator, it has been widely used in military field and civilian field.

In modern war, UAVs with photoelectric reconnaissance system can go deep inside enemy 
territory for reconnaissance,which is the premise of correct command and precision attack, and it's also the important guarantee of the victory in the war. When the plane takes off, flys or lands, it will produce vibration and impact on the moving carrier photoelectric imaging system and affect the image's quality. We reduced the vibration of photoelectric platform by using metal rubber vibration isolator and damped the effects of vibration on imaging quality effectively, so we can obtain accurate and reliable battlefield intelligence.

The torpedo is a kind of water weapon, it plays an important role in antiship and antisubmarine warfare. The torpedo acoustic performance has important influence on the acoustic homing distance, the enemy sonar detection range and the shooting range. In order to improve the fighting capabilities of torpedo and reduce the noise in the torpedo attack, we usually use metal rubber damping element elastic coupling to reduce the coupled vibrations.

Coupled vibrations often happens in the using process of industrial pipes, which seriously influences the performance of the equipment,and also threatens the safety in production. Using metal rubber damping element can effectively solve the problem. The aeroengine pipe clamp and combined clamp of submarines use metal rubber as stretch lining, when they subjected to external load, it dissipates energy through the friction of metal contact point of the spiral coil, thus has the effect of vibration isolation and buffer [5]. So it effectively avoids the coupling vibration of pipeline and improves the stealth performance of submarine.

\section{Expectation}

With the development of our country's economy and the progress of science and technology, the research and application of metal rubber has welcomed a rapid development of historic opportunity. The metal rubber vibration isolator not only has excellent vibration isolation buffer performance, but also has strong ability to adapt the complex and harsh environment. It has a broad application prospect in the field of elastic support of aviation fire control system, ballistic missile instrument compartment, flexible docking structure of large space station and buffer device of alien landing system, and it will play a more and more important role in our national defense and economic construction.

\section{References}

[1] Li Zhong-Ying, The Design of Metal Rubber Components, Beijing: National Defence Industry Press,2000.

[2] Ulanov, A.M, Lauzutkin. Description of an arbitrary multi-axial loading process for non-linear vibration isolations. Journal of Sound and Vibration, 203(5), pp. 903-907,1997.

[3] Li Yu-Yan, Application research on the metal rubber vibration isolator. Process and material, 5(5), pp.62-63,2009.

[4] Yan Hui, Jiang Hong-yuan \& Liu Wen-jian, The parameters identification study of metal rubber vibration isolator with hysteresis nonlinearity. Journal of physics, 58(8), pp.5238-5243,2009.

[5] Jiang Hong-Yuan, Hao De-Gang \& Ao Hong-Rui, System modeling and experimental research of annular metal rubber vibration isolator. Journal of hunan university of science and technology, 20(1), pp.13-16,2005. 